\title{
Treatment of cam-type femoroacetabular impingement
}

\author{
GENNARO FIORENTINO, ALBERTO FONTANAROSA, RICCARDO CEPPARULO, \\ ALBERTO GUARDOLI, LUCA BERNI, GIANLUCA COVIELLO, ALDO GUARDOLI
}

Department of Orthopaedic and Traumatology, Hospital Santa Maria of Borgotaro, Italy

\begin{abstract}
Purpose: the aim of this study was to evaluate preliminary clinical and radiographic results of arthroscopic treatment of cam-type femoroacetabular impingement (FAI).

Methods: thirty-eight patients underwent hip arthroscopy for cam-type FAI between 2009 and 2012. Preoperative assessment was based on clinical examination, modified Harris Hip Score (mHHS) and radiographic examination with anteroposterior pelvis, frogleg and Lequesne views. The patients' clinical conditions at follow-up were assessed using the mHHS administered as a telephone survey. Radiographic outcome measurements evaluated pre and postoperatively were the alpha angle and femoral head-neck offset.

Results: the patients were clinically evaluated at a mean follow-up of 36 months. Radiographic followup was performed at an average of 12.7 months.

Thirty of the 38 patients $(79 \%)$ were satisfied with the results of the arthroscopic procedure. A total of nine patients subsequently underwent a total hip replacement. All 30 patients who declared themselves satisfied recorded an mHHS increase; in particular, the mHHS increased from a mean of 52.9 preoperatively (range: $27.5-82.5$ ) to a mean of 85.6 postoperatively (range: 45.1-100.1). Three significant differences between the two groups of patients (satisfied and not satisfied) were recorded: mean age, alpha angle and BMI were all significantly greater in the patients who were not satisfied with the treatment.
\end{abstract}

\section{Corresponding Author:}

Gennaro Fiorentino, MD

Department of Orthopaedic and Traumatology, Hospital Santa Maria of Borgotaro

Via Benefattori 12, 43043 Borgo Val di Taro, Parma, Italy

E-mail: fiorentino.gennaro2@gmail.com
Conclusions: a crucial aspect in order to obtain good clinical outcomes of arthroscopic treatment of camtype impingement is correct selection of patients who are likely to benefit from this kind of surgery. Hip arthroscopy should be avoided in patients aged over 50 years with risk factors for early osteoarthritis (high BMI and a significantly increased alpha angle).

Level of evidence: Level IV, therapeutic case series.

Key Words: cam-type impingement, hip arthroscopy, alpha angle, femoroplasty.

\section{Introduction}

Cam-type femoroacetabular impingement (FAI) is a known cause of groin pain and a condition that can give rise to osteoarthritis of the hip (1). Patients suffering from this condition are mainly young and experience pain when the hip is moved through internal rotation and adduction at $90^{\circ}$ of hip flexion. In this condition, in predisposed patients, anomalous contact between the femoral head-neck junction and acetabular rim develops. Although FAI had already previously been described as an entity, it was first properly defined and categorized by Ganz et al. (1), who distinguished between different types of FAI: cam, pincer and combined. In cam-type FAI the pathological contact is due to anatomical abnormality of the femur: in this type of FAI, the non-spherical morphology of the femoral head and the subsequent reduction of the femoral head-neck offset are responsible for abnormal contact between the acetabulum and the femur. Stulberg et al. (2) used the term 'pistol-grip' to define this deformity, and according to them it is present in $40 \%$ of patients who develop osteoarthritis of the hip. The cam-type deformity can also be secondary to diseases of the proximal femur typical of childhood, including 
sequelae of slipped capital femoral epiphysis, LeggCalvé-Perthes disease or Perthes-like deformities, and post-infectious and traumatic causes (3).

Femoroacetabular impingement is a dynamic pathology and its development is also related to intense sports activity $(4,5)$. Many patients with FAI may have developed significant secondary joint damage at a younger age when participating in sports, as a result of them pushing their bodies beyond the reduced physiological limits imposed by the altered joint morphology (6). Recent studies have stressed the high prevalence of FAI signs in asymptomatic populations based on previously described cut-off values (7-11). Sutter et al. (9) argue that alpha angle values do not ensure differentiation between the healthy population and affected patients, and suggested that alpha angle cut-off values should be increased. In a study by Lepage-Saucier et al. (7), the $95 \%$ reference interval limits calculated for the signs of FAI were all far beyond the abnormal thresholds found in the literature for cam-type FAI. In a magnetic resonance imaging study, using the predefined cut-off of $50.5,14 \%$ of 200 volunteers had at least one hip with cam morphology (12).

The evidence emerging from the literature suggests that, in anatomically predisposed individuals, alterations in joint morphology (anatomical factors) can develop in relation to how they use their joints (environmental factors), leading to degenerative changes of the articular structures, responsible for the appearance of pain.

Femoroacetabular impingement can be treated with an open surgical procedure (involving dislocation of the hip) or with an arthroscopic procedure. In the case of surgical dislocation clinically relevant complications are reported to occur in $4.8 \%$ of patients. Severe complications are extremely rare but the demanding nature of the surgical procedure, the large incision and the long rehabilitation time mean that this surgery can feasibly be performed only by few operators and centers specializing in hip surgery (13). Otherwise, hip arthroscopy obtains an osseous correction of the cam deformity comparable to the correction obtained with open procedures, and potentially allows smaller incisions, shorter hospitalization, and faster rehabilitation. In addition hip arthroscopy appears to be safe (13). The overall complication rate associated with hip arthroscopy has been reported to be 4.0\% (95 \% CI: 2.9$5.2 \%$ ) with the vast majority of complications being non-life or limb threatening in nature (14). It is well esta- blished in the published literature, which includes a considerable number of patients, that most of the complications related to hip arthroscopy in general are related to patient positioning, traction and fluid management. Adequate padding of compression points on the perineum and the feet is the first step to the achievement of successful hip arthroscopy (15). However, hip arthroscopy is associated with a significant learning curve.

The aim of the present study was to evaluate preliminary clinical and radiographic results of arthroscopic treatment of cam-type FAI. The hypothesis of the study was that arthroscopic treatment of cam-type FAI is an effective and safe procedure.

\section{Methods}

This study was approved by our institutional review board, and all the participants gave their informed consent. Between January 2009 and December 2012, 45 consecutive patients with a clinically and radiographically documented cam-type FAI were treated arthroscopically at our Institute. All the patients underwent a standardized diagnostic assessment, including an interview, physical examination and standard radiographs of the pelvis and hips. The criteria for eligibility to undergo arthroscopic treatment of cam-type FAI (inclusion criteria) were clinical signs of FAI (groin pain, positive anterior impingement test), an alpha angle superior to $50^{\circ}$ on frog-leg radiographs, and a head-neck offset inferior to $8 \mathrm{~mm}$, with a preserved joint space.

\section{Surgical procedure}

The patient was positioned in a supine position on a traction table. Arthroscopy began with visualization of the central compartment where the presence of cam pathology was identified. The presence of labrum failure and degeneration and cartilage joint damage was recorded. Articular degeneration was treated with chondroplasty and microfracture according to its degree. The associated labral pathology was debrided in 14 patients, while in 22 patients the labrum was detached and re-fixed with anchors, both techniques resulting in the creation of a stable edge. The choice between simple debridement or re-fixation of the labrum was made after evaluating the shape, degeneration, tear extension and stability of the labrum with a probe. After concluding the central compartment 
arthroscopy, the cam lesion in the peripheral compartment was treated. A capsulotomy was created by connecting the anterior and anterolateral portals.

The traction was released, and the hip was flexed to approximately $35^{\circ}$ (the traction time was limited to one hour). In this condition the demarcation line between healthy cartilage and the abnormal fibrocartilage that covered the cam lesion can usually be identified. Once the bone was fully exposed, femoroplasty was performed with a spherical $4.5 \mathrm{~mm}$ burr (Figs. 1, 2). In five cases capsulorraphy with a single simple stitch was performed.

\section{Postoperative rehabilitation}

All the patients submitted to a femoroplasty procedure began their rehabilitation with partial weight bearing for 15 days; during this time they wore a hip brace in order to avoid external rotations, extensions and hip flexions over $90^{\circ}$. To prevent adhesions, the hip was mobilized with a continuous passive movement machine from the first day after the operation and with early passive motion with circling movements of the hip. Running was allowed at 12 weeks, while shooting sports could be resumed at 16 weeks. In the patients undergoing microfracture, weight bearing was not allowed for 6-8 weeks.

Indomethacin was prescribed to prevent heterotopic ossification (75 mg twice daily for two weeks).

\section{Postoperative assessment}

The patients were evaluated clinically using the modified Harris Hip Score (mHHS) which was administered pre and postoperatively. We also evaluated the patients' level of satisfaction. The appearance of complications was recorded. The radiographic parameters evaluated, using Philips EBW software (Koninklijke Philips N.V., Eindhoven, The Netherlands) on the pre and postoperative frog-leg radiographs were the alpha angle (Figs. 3, 4) and the femoral head-neck offset.

\section{Results}

Thirty-eight patients were included in the study. Seven patients were excluded because of previous surgery (revision surgery in two cases) or acetabular labral reconstruction with an ileo-tibial band autograft (three cases) or unavailability of correct pre and postoperative radiograph studies (two cases).

The patients were 22 men and 16 women with an average age of 44.4 years. The duration of their symptoms prior to surgical intervention averaged 31 months (range: 4-90 months).

The follow-up clinical evaluation (mHHS, administered as a telephone survey) was performed after a mean interval of 36 months (range: 21-60 months). The radiographic follow-up was obtained after an average of 12.7 months.

Thirty of the 38 patients (79\%) were satisfied (S group) with the results of the arthroscopic procedure; two of these 30 patients subsequently underwent total hip arthroplasty (THR), but they were nevertheless satisfied with the arthroscopic osteochondroplasty as it reduced their pain and delayed THR. Eight patients were not satisfied (NS group) and seven of these also subsequently underwent THR. Three significant diffe-

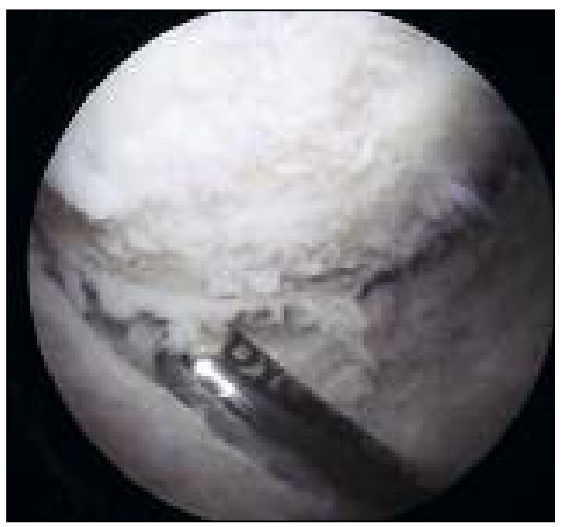

Fig. 1. Intraoperative arthroscopic view of camtype deformity.

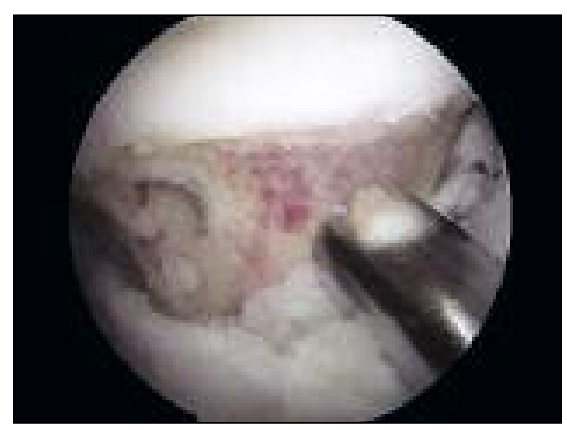

Fig. 2. Intraoperative arthroscopic view of the femoral neck after osteochondroplasty.

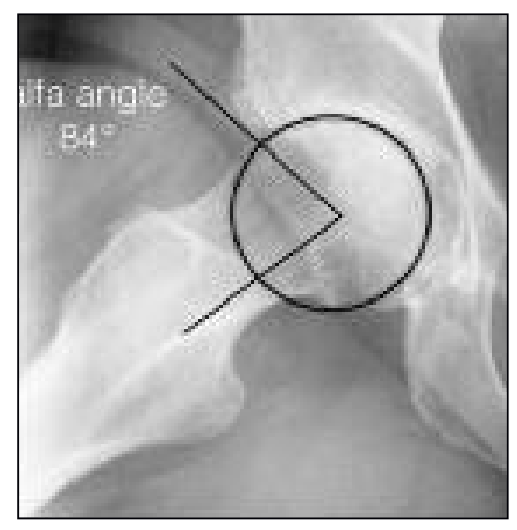

Fig. 3. Preoperative assessment of alpha angle in frog-leg view. 


\section{.

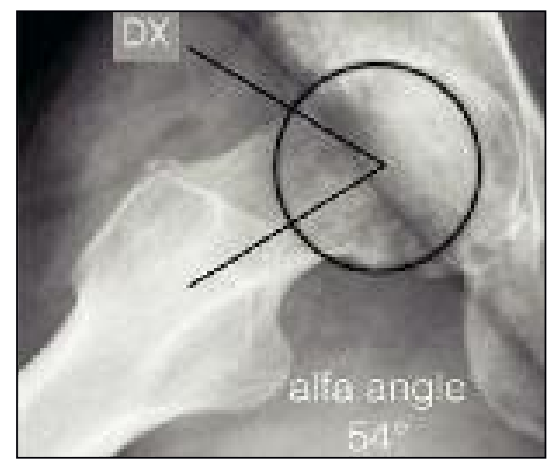

Fig. 4. Postoperative assessment of alpha angle at 6 months of follow-up.

rences between these two groups of patients ( $\mathrm{S}$ and NS) were recorded: a higher mean age in the NS group $(\mathrm{NS}=48.8$ years; $\mathrm{S}=43.3$ years); a greater mean alpha angle in the NS group $\left(\mathrm{NS}=81.2^{\circ} ; \mathrm{S}=73.2^{\circ}\right)$, and a higher body mass index (BMI) in the NS group (NS $=28.3 ; \mathrm{S}=24.7$ ).

The mean mHHS increase from the preoperative period to follow-up was 32.7 points. The mHHS increased in all the $\mathrm{S}$ group patients; in particular mHHS increased from an average of 52.9 preoperatively (range: $27.5-82.5$ ) to an average of 85.6 at followup (range: 45.1-00.1). The pre and postoperative radiographic studies showed a statistically significant change in the mean alpha angle, from $69.7^{\circ}$ preoperatively to $50.7^{\circ}$ at follow-up (a mean reduction of about $19^{\circ}$ ); similarly, we recorded an increase in the head-neck offset, from a mean of $5.44 \mathrm{~mm}$ preoperatively to a mean of $7.76 \mathrm{~mm}$ at follow-up (a mean increase of $2.32 \mathrm{~mm}$ ). Only one complication was observed: a case of pudendal neuropraxia that resolved spontaneously in six months.

\section{Discussion}

Our findings are comparable to those reported in recent systematic reviews evaluating the outcome of hip arthroscopy for FAI (16-18). Such studies have reported patient satisfaction rates of 65 to $85 \%$ at up to 40 months after surgery. We found a patient satisfaction rate of $79 \%$ at follow-up performed at an average of 36 months after arthroscopy. Moreover, the mean age of our cohort was 44.4 years, which is slightly older than in most studies in the literature. A notable finding in our study was the high rate of conversion to arthro- plasty: 9 out of 38 (23.7\%). The mean time to conversion to arthroplasty was 24.3 months after arthroscopy, with a range of 11 to 60 months.

We observed some differences between two groups of patients, those who were not satisfied and those who were satisfied with the procedure; in particular, the NS patients had a higher mean age (48.8 years) compared with the satisfied patients (43.3 years). McCormick et al. (16) suggested that age is a strong predictor of diminished surgical outcome, with worse outcomes in older patients.

The evidence from the literature indicates that surgery gives good results in patients with early degenerative changes of the hip. Philippon et al. (19), in a population of patients aged over 50 years, found that critical factors for poor results were a low preoperative mHHS and a joint space of $2 \mathrm{~mm}$ or less (at 3 years, patients with $2 \mathrm{~mm}$ or less showed a $43 \%$ rate of conversion to THR). In a systematic review by de Sa et al. (20) it was demonstrated that flexion and internal rotation are influenced by the amount of bone correction. An alpha angle correction to $55^{\circ}$ or less is suggested as the target value to obtain good clinical outcomes. However, this applies less in patients with extensive articular damage or advanced osteoarthritis.

Another feature that distinguished the two groups in the present study was the higher mean alpha angle in the NS group. Beaulé et al. (21), in a study of 167 subjects, evaluated radiographic parameters like Wiberg's angle, alpha angle and Tonnis grade on preoperative Xrays and also considered cartilage damage quantified intraoperatively using the Beck's classification. They found that patients with cam-type FAI and an alpha angle of $65^{\circ}$ or more are at increased risk of substantial cartilage damage while increasing acetabular coverage appears to have a protective effect. Other studies indicate that the presence of a cam deformity (as quantified by the alpha angle and head-neck offset) is an independent risk factor for the presence of acetabular cartilage damage and a predictor of the development of osteoarthritis $(22,23)$. Johnston et al. (23) found a significant association between grade of acetabular cartilage defects and degrees of alpha angle. In particular, in hips with grade IV acetabular chondral damage, the alpha angle was $10^{\circ}$ greater than in the hips of patients with no acetabular defects. The alpha angle in patients with large acetabular chondral lesions was $14^{\circ}$ greater than in patients without chondral defects. 
The third characteristic that distinguished between the two groups in the present study was the higher BMI in the NS group. Moderate evidence for a positive association between obesity and the occurrence of hip osteoarthritis has been found (24). In particular, according to recent evidence, BMI should be considered a risk factor for the development of osteoarthritis independently of anatomical abnormalities of the proximal epiphysis of the femur (25).

In conclusion, a crucial aspect, in order to obtain good clinical outcomes of arthroscopic osteochondroplasty for the treatment of Cam-type impingement, is correct selection of patients who are likely to benefit from this kind of surgery. This means that it is necessary to be familiar with the clinical and radiographic parameters that can be considered risk factors for a rapid evolution to hip osteoarthritis. Hip arthroscopy should be avoided in patients aged over 50 years with risk factors for early osteoarthritis (high BMI and a significantly increased alpha angle).

\section{References}

1. Ganz R, Parvizi J, Beck M, et al. Femoroacetabular impingement: a cause for osteoarthritis of the hip. Clin Orthop Relat Res. 2003;417:112-120.

2. Stulberg SD, Cordell LD, Harris WH, et al. Unrecognized childhood hip disease: a major cause of idiopathic osteoarthritis of the hip. In: The Hip. Proc 3rd meeting of The Hip Society. St Louis: CV Mosby Co. 1975;212-228.

3. Siebenrock KA, Schwab JM. The cam-type deformity what is it: SCFE, osteophyte, or a new disease? J Pediatr Orthop. 2013;33 (Suppl 1): S121-125.

4. Siebenrock KA, Ferner F, Noble PC, et al. The cam-type deformity of the proximal femur arises in childhood in response to vigorous sporting activity. Clin Orthop Relat Res. 2011;469: 3229-3240.

5. Lahner M, Walter PA, von Schulze Pellengahr C, et al Comparative study of the femoroacetabular impingement (FAI) prevalence in male semiprofessional and amateur soccer players. Arch Orthop Trauma Surg. 2014;134:1135-1141.

6. Byrd JW, Jones KS. Arthroscopic management of femoroacetabular impingement in athletes. Am J Sports Med. 2011;39 (Suppl):7S-13S.

7. Lepage-Saucier M, Thiéry C, Larbi A, et al. Femoroacetabular impingement: normal values of the quantitative morphometric parameters in asymptomatic hips. Eur Radiol. 2014;24: 1707-1714.

8. Kang ACL, Gooding AJ, Coates MH, et al. Computed tomography assessment of hip joints in asymptomatic individuals in relation to femoroacetabular impingement. Am J Sports Med. 2010;38:1160-1165.

9. Sutter R, Dietrich TJ, Zingg PO, et al. How useful is the alpha angle for discriminating between symptomatic patients with cam-type femoroacetabular impingement and asymptomatic volunteers? Radiology. 2012;264:514-521.

10. Laborie LB, Lehmann TG, Engesæer IØ, et al. Prevalence of radiographic findings thought to be associated with femoroacetabular impingement in a population-based cohort of 2081 healthy young adults. Radiology. 2011;260:494-502.

11. De Bruin F, Reijnierse M, Farhang-Razi V, et al. Radiographic signs associated with femoroacetabular impingement occur with high prevalence at all ages in a hospital population. Eur Radiol. 2013;23:3131-3139.

12. Hack K, Diprimio G, Rakhra K, et al. Prevalence of cam type femoroacetabular impingement in asymptomatic volunteers. J Bone Joint Surg Am. 2010;92A:2436-2444.

13. Buchler L, Neumann M, Schwab JM, et al. Arthroscopic versus open cam resection in the treatment of femoroacetabular impingement. Arthroscopy. 2013;29:653-660.

14. Sink EL, Beaule PE, Sucato D, et al. Multicenter study of complications following surgical dislocation of the hip. J Bone Joint Surg Am. 2011;93A:1132-1136.

15. Kowalczuk M, Bhandari M, Farrokhyar F, et al. Complications following hip arthroscopy: a systematic review and meta-analysis. Knee Surg Sports Traumatol Arthrosc. 2013;21:1669-1675

16. McCormick F, Nwachukwu BU, Alpaugh K, et al. Predictors of hip arthroscopy outcomes for labral tears at minimum 2year follow-up: the influence of age and arthritis. Arthroscopy. 2012;28:1359-1364

17. Bedi A, Chen N, Robertson W, et al. The management of labral tears and femoroacetabular impingement of the hip in the young, active patient. Arthroscopy. 2008;24:1135-1145.

18. Robertson WJ, Kadrmas WR, Kelly BT. Arthroscopic management of labral tears in the hip: a systematic review of the literature. Clin Orthop Relat Res. 2007;455:88-92.

19. Philippon MJ, Souza BG, Briggs KK. Hip arthroscopy for femoroacetabular impingement in patients aged 50 years or older. Arthroscopy. 2012;28:59-65.

20. de Sa D, Urquhart N, Philippon M, et al. Alpha angle correction in femoroacetabular impingement. Knee Surg Sports Traumatol Arthrosc. 2014;22:812-821.

21. Beaulé PE, Hynes K, Parker G, Kemp KA. Can the alpha angle assessment of cam impingement predict acetabular cartilage delamination? Clin Orthop Relat Res. 2012;470:33613367.

22. Anderson LA, Peters CL, Park BB, et al. Acetabular cartilage delamination in femoroacetabular impingement: risk factors and magnetic resonance imaging diagnosis. J Bone Joint Surg Am. 2009;91A:305-313.

23. Johnston TL, Schenker ML, Briggs KK, et al. Relationship between offset angle alpha and hip chondral injury in femoroacetabular impingement. Arthroscopy. 2008;24:669-675.

24. Lievense AM, Bierma-Zeinstra SMA, Verhagen AP, et al. Influence of obesity on the development of osteoarthritis of the hip: a systematic review. Rheumatology (Oxford). 2002;5:1155-1162.

25. Thomas GE, Palmer AJ, Batra RN, et al. Subclinical deformities of the hip are significant predictors of radiographic osteoarthritis and joint replacement in women. A 20 year longitudinal cohort study. Osteoarthritis Cartilage. 2014;22: 1504-1510. 